\title{
The proof of strong Markov property based on one definition (II)
}

\author{
Tang Rong, ${ }^{1, a}$, Liu $\mathrm{Ke}^{2, \mathrm{~b}^{*}}$ and Dong yixuan ${ }^{3, \mathrm{c}}$ \\ ${ }^{1}$ School of Economics and Management, Hainan university, P.R.China,570228 \\ ${ }^{2}$ School of Economics and Management, Hainan university, P.R.China,570228 \\ ${ }^{3}$ logistics management office, Hainan university, P.R.China, 570228 \\ atanyou01@163.com, btangyou01@163.com, 'Dongyx08@163.com
}

Keywords: Markov process, $\sigma$-algebra prior to $\alpha$, Markov property, strong Markov property, stopping time, homogeneity

Abstract. In this paper, on the basis of references [12] we continue to study strong Markov property for general Markov processes. we extend the definition of strong Markov property in [2] to generic Markov processes. and prove them are valid for every Markov processes.

\section{Introduction}

Let $X(t, \omega)$ be a measurable Markov process defining $(\Omega, \boldsymbol{F}, P)$ and valuing $(E, \boldsymbol{E})$. To probability $P\left(\Lambda(\alpha), x_{\alpha+t_{0}} \in A_{0}, x_{\alpha+t_{1}} \in A_{1}\right)$, when $\Lambda(\alpha), A_{0}$ are fixed, $A_{1}$ varies in $\boldsymbol{E}$ to produce a measure on $(E, E)$. Denote as $\bar{P}\left(\Lambda(\alpha), x_{\alpha+t_{0}} \in A_{0}, 9\right.$, that is, for each $A_{1} \in \boldsymbol{E}$

$$
\bar{P}\left(\Lambda(\alpha), x_{\alpha+t_{0}} \in A_{0}, A_{1}\right)=P\left(\Lambda(\alpha), x_{\alpha+t_{0}} \in A_{0}, x_{\alpha+t_{0}} \in A_{1}\right)
$$

In the same reason, probability $P^{\alpha\left(\omega_{0}\right)}\left(\Lambda\left(\alpha\left(\omega_{0}\right)\right), x_{\alpha+t_{0}} \in A_{0}, x_{\alpha+t_{1}} \in A_{1}\right)$ also produce a measure on $(E, \boldsymbol{E})$. Denote as $\bar{P}^{\alpha\left(\omega_{0}\right)}\left(\Lambda\left(\alpha\left(\omega_{0}\right)\right), x_{\alpha+t_{0}} \in A_{0}, 9\right.$, that is, for each $A_{1} \in \boldsymbol{E}$, have

$$
\bar{P}^{\alpha\left(\omega_{0}\right)}\left(\Lambda\left(\alpha\left(\omega_{0}\right)\right), x_{\alpha+t_{0}} \in A_{0}, A_{1}\right)=P^{\alpha\left(\omega_{0}\right)}\left(\Lambda\left(\alpha\left(\omega_{0}\right)\right), x_{\alpha+t_{0}} \in A_{0}, x_{\alpha+t_{1}} \in A_{1}\right)
$$

The above defining measure is applied to definition 1 at once. The following we generalize the definition of strong Markov property in [2] to arbitrary Markov process.

Definition 1. Let $X(t, \omega)$ be a measurable Markov process defining on $(\Omega, \boldsymbol{F}, P)$ and valuing in $(E, \boldsymbol{E}) . P(s, t ; x, A) \quad(0 \leq s<t, x \in E, A \in \boldsymbol{E})$ be its a transition probability function. We call $X(t, \omega)$ has strong Markov property, if $X(t, \omega)$ satisfies:

Let $\alpha(\omega)$ be an arbitrary stopping time, $\boldsymbol{F}_{\alpha} @ \sigma\left(x_{t}: t \leq \alpha\right)$ be the $\sigma$-algebra prior to $\alpha$ generated by $X(t, \omega)$. For each $\Lambda(\alpha) \in \boldsymbol{F}_{\alpha}$, and arbitrary finitely many $0 \leq t_{0}<t_{1}<\mathrm{L}<t_{n} ; A_{0}, A_{1}, \mathrm{~L}, A_{n} \in \boldsymbol{E}$, we have

$$
\begin{aligned}
& P\left(\Lambda(\alpha), x_{\alpha+t_{v}} \in A_{v} ; 0 \leq v \leq n\right)=E\left[\int_{A_{1}} \int_{A_{2}} \mathrm{~L} \int_{A_{n-2}} \int_{A_{n-1}} P\left(\alpha(\omega)+t_{n-1}, \alpha(\omega)+t_{n} ; y_{n-1}, A_{n}\right)\right. \\
& P\left(\alpha(\omega)+t_{n-2}, \alpha(\omega)+t_{n-1} ; y_{n-2}, \mathrm{~d} y_{n-1}\right) P\left(\alpha(\omega)+t_{n-3}, \alpha(\omega)+t_{n-2} ; y_{n-3}, \mathrm{~d} y_{n-2}\right) \mathrm{L} \\
& \left.P\left(\alpha(\omega)+t_{1}, \alpha(\omega)+t_{2} ; y_{1}, \mathrm{~d} y_{2}\right) \bar{P}^{\alpha(\omega)}\left(\Lambda(\alpha(\omega)), x_{\alpha(\omega)+t_{0}} \in A_{0}, \mathrm{~d} y_{1}\right)\right] .
\end{aligned}
$$

In particular, if $X(t, \omega)$ is a homogeneous Markov process, have

$$
\begin{aligned}
& P\left(\Lambda(\alpha), x_{\alpha+t_{v}} \in A_{v} ; 0 \leq v \leq n\right)=\int_{A_{1}} \int_{A_{2}} \mathrm{~L} \int_{A_{n-2}} \int_{A_{n-1}} P\left(t_{n-1}, t_{n} ; y_{n-1}, A_{n}\right) P\left(t_{n-2}, t_{n-1} ; y_{n-2}, \mathrm{~d} y_{n-1}\right) \\
& P\left(t_{n-3}, t_{n-2} ; y_{n-3}, \mathrm{~d} y_{n-2}\right) \mathrm{L} P\left(t_{1}, t_{2} ; y_{1}, \mathrm{~d} y_{2}\right) \bar{P}\left(\Lambda(\alpha), x_{\alpha+t_{0}} \in A_{0}, \mathrm{~d} y_{1}\right) .
\end{aligned}
$$

If $X(t, \omega)$ have strong Markov property, $X(t, \omega)$ is called strong Markov process.

The following conclusion (1) of lemma 1 is the converse theorem of [3, theorem 4.2.1], it is the necessary condition of [3, theorem 4.2.1]. 
Lemma 1. Let $X(t, \omega)$ be a measurable Markov process defining on $(\Omega, \boldsymbol{F}, P)$ and valuing in $(E, \boldsymbol{E}), P(s, t ; x, A) \quad(0 \leq s<t, x \in E, A \in \boldsymbol{E})$ be a transition probability function of $X(t, \omega)$. Then, for arbitrary finitely many $0 \leq t_{0}<t_{1}<\mathrm{L}<t_{n} ; A_{1}, \mathrm{~L}, A_{n} \in \boldsymbol{E}$, we have the following properties:

(1) $P\left(x_{t_{0}} \in A_{0}, x_{t_{1}} \in A_{1}, \mathrm{~L}, x_{t_{n}} \in A_{n}\right)=$

$$
\int_{A_{0}} \int_{A_{1}} \mathrm{~L} \int_{A_{n-1}} P\left(t_{n-1} t_{n} ; y_{n-1}, A_{n}\right) P\left(t_{n-2}, t_{n-1} ; y_{n-2}, \mathrm{~d} y_{n-1}\right) \mathrm{L} P\left(t_{0}, t_{1} ; y_{0}, \mathrm{~d} y_{1}\right) P_{t_{0}}\left(\mathrm{~d} y_{0}\right)
$$

where $P_{t_{0}}\left(g\right.$ is a probability measure on $(E, \boldsymbol{E})$ derived by $x_{t_{0}}$, that is, $P_{t_{0}}(A) @ P\left(x_{t_{0}} \in A\right), A \in \boldsymbol{E}$.

(2) $P\left(x_{t_{1}} \in A_{1}, \mathrm{~L}, x_{t_{n}} \in A_{n} \mid \sigma\left(x_{t_{0}}\right)\right)(\omega)=$

$$
\left.\int_{A_{1}} \mathrm{~L} \int_{A_{n-1}} P\left(t_{n-1}, t_{n} ; y_{n-1}, A_{n}\right) P\left(t_{n-2}, t_{n-1} ; y_{n-2}, \mathrm{~d} y_{n-1}\right) \mathrm{L} P\left(t_{0}, t_{1} ; x_{t_{0}}(\omega), \mathrm{d} y_{1}\right), \quad \mathrm{P}_{\sigma\left(\mathrm{x}_{\mathrm{t}_{0}}\right.}\right) \text {-a.e.. }
$$

Particularly take $n=2, A_{1}=E$, and $P\left(t_{0}, t_{2} ; x_{t_{0}}(\omega), A_{2}\right)$ replace $P\left(x_{t_{2}} \in A_{2} \mid \sigma\left(x_{t_{0}}\right)\right)(\omega)$ ( Because $P\left(t_{0}, t_{2} ; x_{t_{0}}(\omega), A_{2}\right)$ is a version of $\left.P\left(x_{t_{2}} \in A_{2} \mid \sigma\left(x_{t_{0}}\right)\right)(\omega)\right)$. Then above equation is changed into K-C equation:

$$
\left.P\left(t_{0}, t_{2} ; x_{t_{0}}(\omega), A_{2}\right)=\int_{E} P\left(t_{1}, t_{2} ; y_{1}, A_{2}\right) P\left(t_{0}, t_{1} ; x_{t_{0}}(\omega), \mathrm{d} y_{1}\right), \quad \mathrm{P}_{\sigma\left(\mathrm{x}_{\mathrm{t}_{0}}\right.}\right) \text {-a.e.. }
$$

Proof. (1). We apply inductive method to prove this conclusion. For $n=1$,

$$
\begin{aligned}
& P\left(x_{t_{0}} \in A_{0}, x_{t_{1}} \in A_{1}\right)=\int_{\left\{x_{t_{0}} \in A_{0}\right\}} P\left(x_{t_{1}} \in A_{1} \mid \sigma\left(x_{t_{0}}\right)\right)(\omega) P(\mathrm{~d} \omega)=\int_{\left\{x_{t_{0}} \in A_{0}\right\}} P\left(t_{0}, t_{1} ; x_{t_{0}}(\omega), A_{1}\right) P(\mathrm{~d} \omega) \\
& =\int_{A_{0}} P\left(t_{0}, t_{1} ; y_{0}, A_{1}\right) P_{t_{0}}\left(\mathrm{~d} y_{0}\right), \text { by the theorem of integral transformation. }
\end{aligned}
$$

Suppose that for $n-1$ the conclusion holds, that is, $P\left(x_{t_{0}} \in A_{0}, x_{t_{1}} \in A_{1}, \mathrm{~L}, x_{t_{n-1}} \in A_{n-1}\right)=$ $\int_{A_{0}} \int_{A_{1}} \mathrm{~L} \int_{A_{n-2}} P\left(t_{n-2}, t_{n-1} ; y_{n-2}, A_{n-1}\right) P\left(t_{n-3}, t_{n-2} ; y_{n-3}, \mathrm{~d} y_{n-2}\right) \mathrm{L} P\left(t_{0}, t_{1} ; y_{0}, \mathrm{~d} y_{1}\right) P_{t_{0}}\left(\mathrm{~d} y_{0}\right)$.

Fix $A_{0}, A_{1}, \mathrm{~L}, A_{n-2}$, but $A_{n-1}$ vary in $\boldsymbol{E}$. Both the left term and the right term above equality are measures on $(E, \boldsymbol{E})$. denote by $\bar{P}\left(A_{0}, A_{1}, \mathrm{~L}, A_{n-2}, 9\right.$ and $\bar{\mu}\left(A_{0}, A_{1}, \mathrm{~L}, A_{n-2}, 9\right.$ respectively. By the inductive assumption, $\bar{P}\left(A_{0}, A_{1}, \mathrm{~L}, A_{n-2}, \mathrm{~g}=\bar{\mu}\left(A_{0}, A_{1}, \mathrm{~L}, A_{n-2}, \mathrm{~g}\right.\right.$. If $\left\{x_{t_{n-1}} \in A_{n-1}\right\}$ is substituted into $\bar{P}\left(A_{0}, A_{1}, \mathrm{~L}, A_{n-2}, \mathrm{~g}\right.$, The measure on $\left(\Omega, \sigma\left(x_{t_{n-1}}\right)\right)$ is obtained. denoted by $P\left(A_{0}, A_{1}, \mathrm{~L}, A_{n-2}, 9\right.$. So, we have $\bar{P}\left(A_{0}, A_{1}, \mathrm{~L}, A_{n-2}, A_{n-1}\right)=P\left(A_{0}, A_{1}, \mathrm{~L}, A_{n-2},\left\{x_{t_{n-1}} \in A_{n-1}\right\}\right)$. Hence,

$$
P\left(x_{t_{0}} \in A_{0}, x_{t_{1}} \in A_{1}, \mathrm{~L}, x_{t_{n}} \in A_{n}\right)=\int_{\left\{x_{t_{0}} \in A_{0}, \mathrm{~L}, x_{t_{n-1}} \in A_{n-1}\right\}} P\left(x_{t_{n}} \in A_{n} \mid \sigma\left(x_{t_{0}}, \mathrm{~L}, x_{t_{n-1}}\right)\right) P(\mathrm{~d} \omega)
$$

$=\int_{\left\{x_{t 0} \in A_{0}, \mathrm{~L}, x_{t_{n-1}} \in A_{n-1}\right\}} P\left(x_{t_{n}} \in A_{n} \mid \sigma\left(x_{t_{n-1}}\right)\right) P(\mathrm{~d} \omega)$, by Markov property,

$=\int_{\left\{x_{t_{0}} \in A_{0}, \mathrm{~L}, x_{t n-1} \in A_{n-1}\right\}} P\left(t_{n-1}, t_{n} ; x_{t_{n-1}}(\omega), A_{n}\right) P(\mathrm{~d} \omega)$, since $P\left(x_{t_{n}} \in A_{n} \mid \sigma\left(x_{t_{n-1}}\right)\right)=P\left(t_{n-1}, t_{n} ; x_{t_{n-1}}(\omega), A_{n}\right)$, P-a.e.,

$=\int_{\left\{x_{n-1} \in A_{n-1}\right\}} P\left(t_{n-1}, t_{n} ; x_{t_{n-1}}(\omega), A_{n}\right) P\left(A_{0}, A_{1}, \mathrm{~L}, A_{n-2}, \mathrm{~d} \omega\right)$, by integral transformation,

$=\int_{A_{n-1}} P\left(t_{n-1}, t_{n} ; x_{t_{n-1}}(\omega), A_{n}\right) \bar{P}\left(A_{0}, A_{1}, \mathrm{~L}, A_{n-2}, \mathrm{~d} y_{n-1}\right)$, by integral transformation,

$=\int_{A_{n-1}} P\left(t_{n-1}, t_{n} ; x_{t_{n-1}}(\omega), A_{n}\right) \bar{\mu}\left(A_{0}, A_{1}, \mathrm{~L}, A_{n-2}, \mathrm{~d} y_{n-1}\right)$, by inductive assumption,

$=\int_{A_{0}} \int_{A_{1}} \mathrm{~L} \int_{A_{n-1}} P\left(t_{n-1} t_{n} ; y_{n-1}, A_{n}\right) P\left(t_{n-2}, t_{n-1} ; y_{n-2}, \mathrm{~d} y_{n-1}\right) \mathrm{L} P\left(t_{0}, t_{1} ; y_{0}, \mathrm{~d} y_{1}\right) P_{t_{0}}\left(\mathrm{~d} y_{0}\right)$.

Remark 1. From third to sixth equalities also can obtain by similar to the proof of [12, (5)].

(2). By (1) and the theorem of integral transformation,

$$
\begin{aligned}
& \int_{\left\{x_{t_{0}} \in A_{0}\right\}} \int_{A_{1}} \mathrm{~L} \int_{A_{n-1}} P\left(t_{n-1} t_{n} ; y_{n-1}, A_{n}\right) P\left(t_{n-2}, t_{n-1} ; y_{n-2}, \mathrm{~d} y_{n-1}\right) \mathrm{L} P\left(t_{0}, t_{1} ; y_{0}, \mathrm{~d} y_{1}\right) P(\mathrm{~d} \omega) \\
& =\int_{A_{0}} \int_{A_{1}} \mathrm{~L} \int_{A_{n-1}} P\left(t_{n-1} t_{n} ; y_{n-1}, A_{n}\right) P\left(t_{n-2}, t_{n-1} ; y_{n-2}, \mathrm{~d} y_{n-1}\right) \mathrm{L} P\left(t_{0}, t_{1} ; y_{0}, \mathrm{~d} y_{1}\right) P_{t_{0}}\left(\mathrm{~d} y_{0}\right) \\
& =P\left(x_{t_{0}} \in A_{0}, x_{t_{1}} \in A_{1}, \mathrm{~L}, x_{t_{n}} \in A_{n}\right)=\int_{\left\{x_{t_{0}} \in A_{0}\right\}} P\left(x_{t_{1}} \in A_{1}, \mathrm{~L}, x_{t_{n}} \in A_{n} \mid \sigma\left(x_{t_{0}}\right)\right)(\omega) P(\mathrm{~d} \omega)
\end{aligned}
$$


So by Radon-Nikodym's theorem yields (2).

Theorem 1. Let $X(t, \omega)$ be an arbitrary measurable Markov process defining on $(\Omega, \boldsymbol{F}, P)$ and valuing in $(E, \boldsymbol{E}) . P(s, t ; x, A) \quad(0 \leq s<t, x \in E, A \in \boldsymbol{E})$ be one transition probability function of $X(t, \omega)$. Then $X(t, \omega)$ is a strong Markov process.

\section{Proof. First Let}

$\Lambda(\alpha)=\left\{x_{s_{1}} \in B_{1}, \mathrm{~L}, x_{s_{m}} \in B_{m}, \alpha \geq s_{m+1}\right\}$, where $m \geq 1 ; 0 \leq s_{1} \leq \mathrm{L} \leq s_{m+1} \leq \infty ; B_{1}, \mathrm{~L}, B_{m} \in \boldsymbol{E}$.

Then,

$P\left(\Lambda(\alpha), x_{\alpha+t_{v}} \in A_{v} ; 0 \leq v \leq n\right)=$

$\int_{\Omega} \int_{\left\{\Lambda\left(\alpha\left(\omega_{0}\right)\right), x_{\alpha\left(\omega_{0}\right)+t_{0}} \in A_{0}\right\}} P\left(x_{\alpha\left(\omega_{0}\right)+t_{v}} \in A_{v} ; 1 \leq v \leq n \mid \sigma\left(x_{\alpha\left(\omega_{0}\right)+t_{0}}\right)\right) P^{\alpha\left(\omega_{0}\right)}(\mathrm{d} \omega) P\left(\mathrm{~d} \omega_{0}\right)$, which follows from the same as proof of [12, theorem 1$]$,

$=\int_{\Omega} \int_{\left\{\Lambda\left(\alpha\left(\omega_{0}\right)\right), x_{\alpha\left(\omega_{0}\right)+10} \in A_{0}\right\}}\left[\int_{A_{1}} \mathrm{~L} \int_{A_{n-1}} P\left(\alpha\left(\omega_{0}\right)+t_{n-1}, \alpha\left(\omega_{0}\right)+t_{n} ; y_{n-1}, A_{n}\right) P\left(\alpha\left(\omega_{0}\right)+t_{n-2}, \alpha\left(\omega_{0}\right)+t_{n-1}\right.\right.$

$\left.\left.; y_{n-2}, \mathrm{~d} y_{n-1}\right) \mathrm{L} P\left(\alpha\left(\omega_{0}\right)+t_{0}, \alpha\left(\omega_{0}\right)+t_{1} ; x_{\alpha\left(\omega_{0}\right)+t_{0}}(\omega), \mathrm{d} y_{1}\right)\right] P^{\alpha\left(\omega_{0}\right)}(\mathrm{d} \omega) P\left(\mathrm{~d} \omega_{0}\right)$, by lemma 1 ,

$=\int_{\Omega} \int_{A_{1}} \mathrm{~L} \int_{A_{n-1}} P\left(\alpha\left(\omega_{0}\right)+t_{n-1}, \alpha\left(\omega_{0}\right)+t_{n} ; y_{n-1}, A_{n}\right) P\left(\alpha\left(\omega_{0}\right)+t_{n-2}, \alpha\left(\omega_{0}\right)+t_{n-1} ; y_{n-2}, \mathrm{~d} y_{n-1}\right) \mathrm{L} P\left(\alpha\left(\omega_{0}\right)+t_{1}\right.$,

$\left.\left.\alpha\left(\omega_{0}\right)+t_{2} ; y_{1}, \mathrm{~d} y_{2}\right)\left[\int_{\left\{\Lambda\left(\alpha\left(\omega_{0}\right)\right), x_{\alpha\left(\omega_{0}\right)+10} \in A_{0}\right\}} P\left(\alpha\left(\omega_{0}\right)+t_{0}, \alpha\left(\omega_{0}\right)+t_{1} ; x_{\alpha\left(\omega_{0}\right)+t_{0}}(\omega), \mathrm{d} y_{1}\right)\right] P^{\alpha\left(\omega_{0}\right)}(\mathrm{d} \omega)\right] P\left(\mathrm{~d} \omega_{0}\right)$

,exchange the integral sequence,

$=\int_{\Omega}\left[\int_{A_{1}} \mathrm{~L} \int_{A_{n-1}} P\left(\alpha\left(\omega_{0}\right)+t_{n-1}, \alpha\left(\omega_{0}\right)+t_{n} ; y_{n-1}, A_{n}\right) P\left(\alpha\left(\omega_{0}\right)+t_{n-2}, \alpha\left(\omega_{0}\right)+t_{n-1} ; y_{n-2}, \mathrm{~d} y_{n-1}\right) \mathrm{L}\right.$

$\left.P\left(\alpha\left(\omega_{0}\right)+t_{1}, \alpha\left(\omega_{0}\right)+t_{2} ; y_{1}, \mathrm{~d} y_{2}\right) \bar{P}^{\alpha\left(\omega_{0}\right)}\left(\Lambda\left(\alpha\left(\omega_{0}\right)\right), x_{\alpha\left(\omega_{0}\right)+t_{0}} \in A_{0}, \mathrm{~d} y_{1}\right)\right] P\left(\mathrm{~d} \omega_{0}\right)$

$=E\left[\int_{A_{1}} \mathrm{~L} \int_{A_{n-1}} P\left(\alpha\left(\omega_{0}\right)+t_{n-1}, \alpha\left(\omega_{0}\right)+t_{n} ; y_{n-1}, A_{n}\right) P\left(\alpha\left(\omega_{0}\right)+t_{n-2}, \alpha\left(\omega_{0}\right)+t_{n-1} ; y_{n-2}, \mathrm{~d} y_{n-1}\right) \mathrm{L}\right.$

$\left.P\left(\alpha\left(\omega_{0}\right)+t_{1}, \alpha\left(\omega_{0}\right)+t_{2} ; y_{1}, \mathrm{~d} y_{2}\right) \bar{P}^{\alpha\left(\omega_{0}\right)}\left(\Lambda\left(\alpha\left(\omega_{0}\right)\right), x_{\alpha\left(\omega_{0}\right)+t_{0}} \in A_{0}, \mathrm{~d} y_{1}\right)\right]$

where the (2) make use of the following equality

$$
\begin{gathered}
\left.\int_{\left\{\Lambda\left(\alpha\left(\omega_{0}\right)\right), x_{\alpha\left(\omega_{0}\right)+t_{0}} \in A_{0}\right\}} P\left(\alpha\left(\omega_{0}\right)+t_{0}, \alpha\left(\omega_{0}\right)+t_{1} ; x_{\alpha\left(\omega_{0}\right)+t_{0}}(\omega), \mathrm{d} y_{1}\right)\right] P^{\alpha\left(\omega_{0}\right)}(\mathrm{d} \omega)= \\
P^{\alpha\left(\omega_{0}\right)}\left(\Lambda\left(\alpha\left(\omega_{0}\right)\right), x_{\alpha\left(\omega_{0}\right)+t_{0}} \in A_{0}, x_{\alpha\left(\omega_{0}\right)+t_{1}} \in A_{1}\right) .
\end{gathered}
$$

In fact, above formula is obtained by [12, lemma 2]. In particular, if $X(t, \omega)$ is a homogeneous Markov process, by the homogeneity we know (2) is changed into

$$
\begin{aligned}
P & \left(\Lambda(\alpha), x_{\alpha+t_{v}} \in A_{v} ; 0 \leq v \leq n\right) \\
= & \int_{\Omega}\left[\int_{A_{1}} \mathrm{~L} \int_{A_{n-1}} P\left(t_{n-1}, t_{n} ; y_{n-1}, A_{n}\right) P\left(t_{n-2}, t_{n-1} ; y_{n-2}, \mathrm{~d} y_{n-1}\right) \mathrm{L} P\left(t_{1}, t_{2} ; y_{1}, \mathrm{~d} y_{2}\right)\right. \\
& \left.\bar{P}^{\alpha\left(\omega_{0}\right)}\left(\Lambda\left(\alpha\left(\omega_{0}\right)\right), x_{\alpha\left(\omega_{0}\right)+t_{0}} \in A_{0}, \mathrm{~d} y_{1}\right)\right] P\left(\mathrm{~d} \omega_{0}\right) \\
= & \int_{A_{1}} \mathrm{~L} \int_{A_{n-1}} P\left(t_{n-1}, t_{n} ; y_{n-1}, A_{n}\right) P\left(t_{n-2}, t_{n-1} ; y_{n-2}, \mathrm{~d} y_{n-1}\right) \mathrm{L} P\left(t_{1}, t_{2} ; y_{1}, \mathrm{~d} y_{2}\right) \\
& {\left[\int_{\Omega} \bar{P}^{\alpha\left(\omega_{0}\right)}\left(\Lambda\left(\alpha\left(\omega_{0}\right)\right), x_{\alpha\left(\omega_{0}\right)+t_{0}} \in A_{0}, \mathrm{~d} y_{1}\right) P\left(\mathrm{~d} \omega_{0}\right)\right] } \\
= & \int_{A_{1}} \mathrm{~L} \int_{A_{n-1}} P\left(t_{n-1}, t_{n} ; y_{n-1}, A_{n}\right) P\left(t_{n-2}, t_{n-1} ; y_{n-2}, \mathrm{~d} y_{n-1}\right) \mathrm{L} P\left(t_{1}, t_{2} ; y_{1}, \mathrm{~d} y_{2}\right) \bar{P}\left(\Lambda(\alpha), x_{\alpha+t_{0}} \in A_{0}, \mathrm{~d} y_{1}\right), \quad \text { by }
\end{aligned}
$$

the definition of conditional probability and [12, lemma 5].

So by $\lambda-\pi$-system method it follows this theorem.

Remark 2. For above multiple integral, we may start from inside to outside, this is the essential convention of multiple integral. also may start from outside to inside, From the proof of lemma 1 and basic idea of induction we know it is valid. In the end, we point out the associative law may be arbitrarily used to above multiple integral, that is, exchange the integral sequence is valid. We explain it by the following example:

$$
\int_{A_{0}} \int_{A_{1}} \int_{A_{2}} \int_{A_{3}} P\left(t_{3}, t_{4} ; y_{3}, A_{4}\right) P\left(t_{2}, t_{3} ; y_{2}, \mathrm{~d} y_{3}\right) P\left(t_{1}, t_{2} ; y_{1}, \mathrm{~d} y_{2}\right) P\left(t_{0}, t_{1} ; x_{t_{0}}(\omega), \mathrm{d} y_{1}\right) P(\mathrm{~d} \omega) \text {. }
$$


If $A_{1}$ fix, and $A_{2}$ vary in $\boldsymbol{E}, P^{x_{t_{0}}(\omega)}\left(x_{t_{1}} \in A_{1}, x_{t_{2}} \in A_{2}\right)$ is changed into a measure defining on $\boldsymbol{E}$. Denote by $\bar{P}^{x_{t 0}(\omega)}\left(x_{t_{1}} \in A_{1}, g\right.$. By lemma $1(2)$,

$\int_{A_{1}} P\left(t_{1}, t_{2} ; y_{1}, A_{2}\right) P\left(t_{0}, t_{1} ; x_{t_{0}}(\omega), \mathrm{d} y_{1}\right)=\bar{P}^{x_{t_{0}}(\omega)}\left(x_{t_{1}} \in A_{1}, A_{2}\right), \quad \mathrm{P}_{\sigma\left(x_{t_{0}}\right)}$-a.e..

So, similar to the proof of (5) in reference [12] it follows

$\int_{A_{0}} \int_{A_{1}} \int_{A_{2}} \int_{A_{3}} P\left(t_{3}, t_{4} ; y_{3}, A_{4}\right) P\left(t_{2}, t_{3} ; y_{2}, \mathrm{~d} y_{3}\right) P\left(t_{1}, t_{2} ; y_{1}, \mathrm{~d} y_{2}\right) P\left(t_{0}, t_{1} ; x_{t_{0}}(\omega), \mathrm{d} y_{1}\right) P(\mathrm{~d} \omega)$

$=\int_{A_{0}} \int_{A_{2}} \int_{A_{3}} P\left(t_{3}, t_{4} ; y_{3}, A_{4}\right) P\left(t_{2}, t_{3} ; y_{2}, \mathrm{~d} y_{3}\right) \bar{P}^{x_{t_{0}}(\omega)}\left(x_{t_{1}} \in A_{1}, \mathrm{~d} y_{2}\right) P(\mathrm{~d} \omega)$

$=\int_{A_{0}} \int_{A_{2}} \int_{A_{3}} P\left(t_{3}, t_{4} ; y_{3}, A_{4}\right) P\left(t_{2}, t_{3} ; y_{2}, \mathrm{~d} y_{3}\right)\left[\int_{A_{1}} P\left(t_{1}, t_{2} ; y_{1}, \mathrm{~d} y_{2}\right) P\left(t_{0}, t_{1} ; x_{t_{0}}(\omega), \mathrm{d} y_{1}\right) P(\mathrm{~d} \omega)\right] P(\mathrm{~d} \omega)$

$=\int_{A_{0}}\left[\int_{A_{1}}\right] \int_{A_{2}} \int_{A_{3}} P\left(t_{3}, t_{4} ; y_{3}, A_{4}\right) P\left(t_{2}, t_{3} ; y_{2}, \mathrm{~d} y_{3}\right) P\left(t_{1}, t_{2} ; y_{1}, \mathrm{~d} y_{2}\right) P\left(t_{0}, t_{1} ; x_{t_{0}}(\omega), \mathrm{d} y_{1}\right) P(\mathrm{~d} \omega)$

Which is the rationale of that (1) holds.

\section{References}

[1] Yan Shijian etc.. The foundation of probability[M].Science Press, Beijing, 2007. (in Chinese)

[2] Zikun Wang, Xiangqun Yang. Birth and death Processes and Markov chains. Science Press, Beijing, 2006.

[3] Wang Zikun. The general theory of stochastic process[M], Beijing Normal University Press, Beijing, 1996. (in Chinese)

[4] Qian Mingpin, Gong Guanglu. The theory stocastic processes[M], Peking University Press, Beijing, 1997. (in Chinese)

[5] Wan Chenggao. The limit theory of martingale, Science Press, Beijing, 2002. (in Chinese)

[6] Daniel Ray. Stationary Markov processes with continuous paths[J], Trans. Amer. Math. Soc. Vol. 82 (1956), pp. 452-493.

[7] Tang Rong, Huang Yonghui. The truncated Markov processes prior to $\alpha$ and the truncated Markov processes after $\alpha[\mathrm{J}]$, Acta scientiarum naturalium universitatis sunyatseni, Guang Zhou, 2009, Vol. 48, No.2, pp. 5-10. (in Chinese)

[8] Yan Jiaan, The theory of martingale and stochastic integral, Shanghai Science and Technology Press, Shanghai, 1981. (in Chinese)

[9] Tang Rong, Huang Yonghui. The research on the strong Markov property[J]. Annales universitatis paedagogicae cracoviensis studia mathematica, 2011, Vol. 10, pp. 35-65 ( http://studmath.up.krakow.pl )

[10] Robert B.Ash and Catherine A. Doléans-Dade, Porbability \& measurable theory, (print in china), Post \& telecom press, Beijing, 2007.

[11] Tang Rong. Annotations of two examples about Markov process[J]. Journal of mathematical sciences: advances and applications, 2013, Vol. 21, pp 17-38. (http://scientificadvances.co.in)

[12] R. Tang, Y. X. Dong, The Proof of Strong Markov Property Based on one Definition[A], in: 5th International Conference on Advances in Materials and Manufacturing Processes[C]. Switzerland: Applied Mechanics and Materials, Vol. 742, Mar. 2015, pp. 419-428. 